\title{
Psicologia da Saúde e Cronobiologia: Diálogo Possível?
}

Health Psychology And Chronobiology: Is A Dialogue Possible?

Psicología De La Salud Y Cronobiología: ¿Diálogo Posible?

Katie Moraes de

Almondes

Universidade Federal do Rio Grande do

Norte
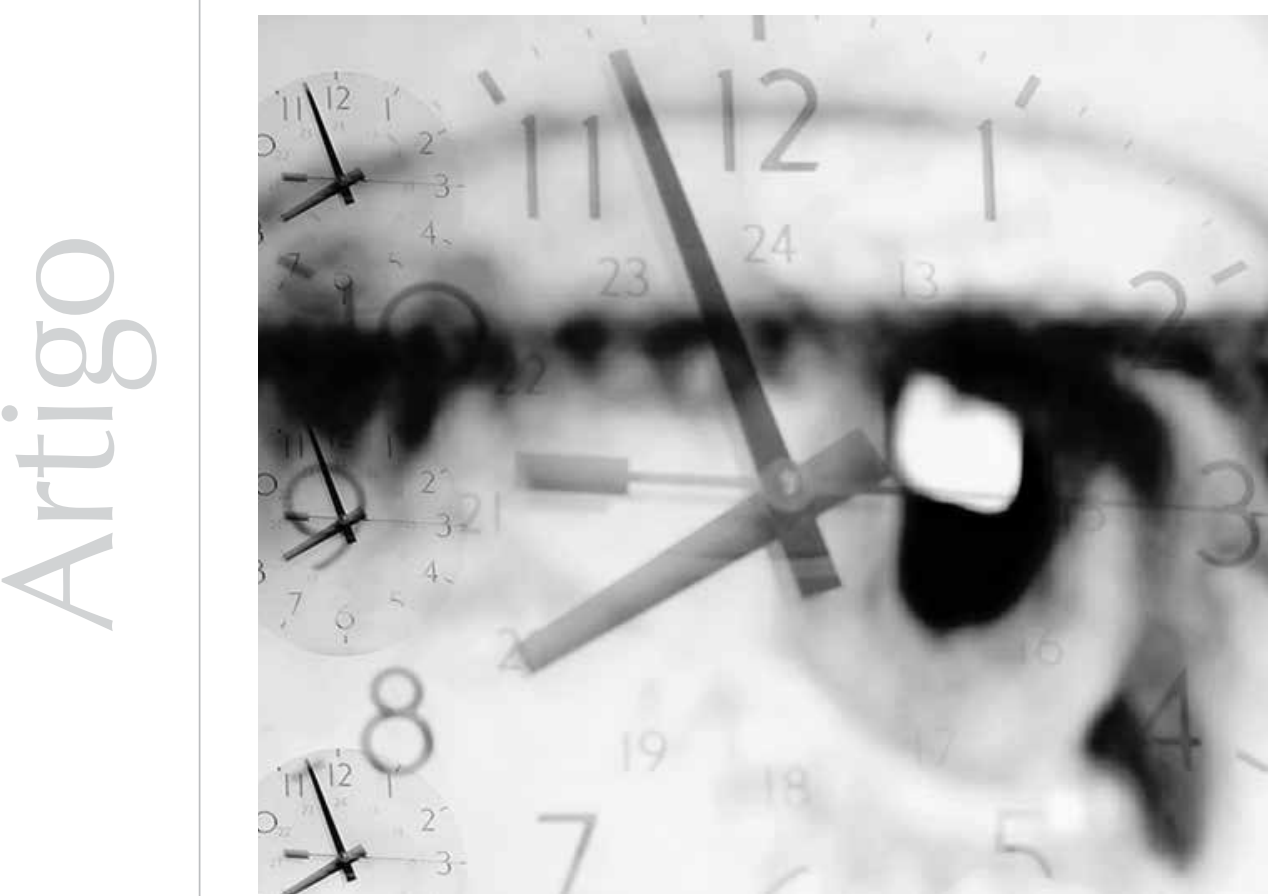
Resumo: A Psicologia da saúde visa a avaliar o papel do comportamento na etiologia da doença, a elaborar prognósticos dos comportamentos prejudiciais à saúde e a promover comportamentos saudáveis. Seu pressuposto epistemológico, o modelo biopsicossocial, envolve a interação de fatores biológicos, psicológicos e sociais na análise do processo saúde e doença. A cronobiologia ocupa-se da investigação temporal recorrente de variáveis fisiológicas e comportamentais assim como do ajuste temporal dessas variáveis ao ambiente e às suas demandas sociais. A proposta deste artigo, de caráter teórico, foi expor a interseção entre essas duas áreas, evidenciando que ambas contribuem para a compreensão dos efeitos biopsicossociais na saúde, para a identificação precoce de pessoas em situação de risco e para a prevenção de comportamentos saudáveis a fim de enfrentar situações conflituosas, além do seu papel na elaboração de programas de promoção à saúde, de estudos e de intervenções no campo comunitário e na saúde pública. São fundamentalmente especialidades interdisciplinares, que envolvem conhecimentos fisiológicos, comportamentais, cognitivos, educativos e ambientais e que fazem com que essa interseção se consolide. Palavras-chave: Psicologia da saúde. Cronobiologia. Comportamento. Cognição.

\begin{abstract}
Health psychology evaluates the role of behavior in the etiology of disease, develops predictions of behaviors that are harmful to health, and promotes healthy behaviors. Its epistemological assumption, the biopsychosocial model, involves the interaction of biological, psychological and social analysis of health and disease. Chronobiology is concerned with the investigation of recurrent temporal physiological and behavioral variables as well as the temporal adjustment of these variables to the environment and their social demands. The purpose of this theoretical article was to expose the intersection between these two areas showing that both contribute for the understanding of the biopsychosocial effects on health, for the early identification of those at risk and for the prevention of healthy behaviors to deal with conflicting situations, in addition to its role in development programs of health promotion, studies and interventions in the community and public health. These specialties are fundamentally interdisciplinary, involving physiological, behavioral, cognitive, educational and environmental knowledge, making this intersection consolidated.
\end{abstract}

Keywords: Health care psychology. Chronobiology. Behavior. Cognition.

Resumen: La psicología de la salud visa evaluar el papel del comportamiento en la etiología de la enfermedad, elaborar pronósticos de los comportamientos prejudiciales a la salud e incentivar comportamientos saludables. Su presupuesto epistemológico, el modelo biopsicosocial, involucra la interacción de factores biológicos, psicológicos y sociales en el análisis del proceso salud y enfermedad. La cronobiología se ocupa de la investigación temporal consecuencia de variables fisiológicas y comportamentales así como del ajuste temporal de esas variables al ambiente y a sus demandas sociales. La propuesta de este artículo, de carácter teórico, fue exponer la intersección entre esas dos áreas, evidenciando que ambas contribuyen para la comprensión de los efectos biopsicosociales en la salud, para la identificación precoz de personas en situación de riesgo y para la prevención de comportamientos saludables con el fin de enfrentar situaciones conflictivas, además de su papel en la elaboración de programas de promoción a la salud, de estudios y de intervenciones en el campo comunitario y en la salud pública. Son fundamentalmente especialidades interdisciplinarias, que involucran conocimientos fisiológicos, comportamentales, cognitivos, educativos y ambientales y que hacen que esa intersección se consolide.

Palabras clave: Psicología de la salud. Cronobiología. Comportamiento. Cognición.

A Psicologia da saúde é o mais recente desenvolvimento da inserção da ciência psicológica no campo de compreensão da saúde, e antecede a Medicina comportamental, a Medicina psicossomática e a saúde comportamental. Essa área constitui um modelo opositor do ideário do curativismo, do mecanicismo e da noção de unicausalidade (motores do modelo biomédico - Westphal \& Santos, 1999), que avigoram a ideia de que a saúde é a ausência de doença, e, dessa forma, as práticas em saúde têm como função principal a cura das doenças físicas, desconsiderando que os indivíduos podem ser responsáveis pelo seu estado de saúde devido a suas crenças e a seus comportamentos de risco, pelas suas estratégias de coping inadequadas e pela sua não adesão às recomendações médicas.

A Psicologia da saúde formalmente remonta à década de 70, mais exatamente a 1973, com a criação de uma Task Force on Health Research pela American Psychological Association (APA, 1976), cujo objetivo foi desenvolver pesquisas básicas e aplicadas 
sobre o papel do comportamento nas doenças físicas. Em 1978, a APA institui a Divisão de Psicologia da Saúde, conhecida como a Divisão 38, conceituando a Psicologia da saúde como contribuições da Psicologia para: avaliações do papel do comportamento na etiologia e no tratamento da doença, elaboração de prognósticos dos comportamentos prejudiciais para a saúde, promoção de comportamentos saudáveis e prevenção do aparecimento das doenças (American Psychological Association - APA, 2011). Mas essa área da Psicologia já era pauta de discussões relativas a intervenções, no Ministério da Saúde de Cuba, no final dos anos 60, época em que foi inaugurada a Sociedade Cubana de Psicologia da Saúde.

Após esses marcadores históricos e com a evolução da área, Stone (1979 como citado na APA, 2011) é um dos primeiros a conceituar a Psicologia da saúde chamando a atenção para uma psicologia que tinha um plano metodológico aplicável a todas as situações próprias do campo da saúde, que envolvia desde a educação até a saúde pública, os cuidados e a legislação. Classicamente, é a definição de Matarazzo (1980), que absorveu a fonte da definição da APA e de Stone, que é lembrada, tendo sido acrescentado o papel dos psicólogos na discussão do aperfeiçoamento do sistema político de saúde, que afirma que a Psicologia da saúde constitui estratégia de atuação que agrega o conhecimento educacional, científico e profissional da Psicologia para as discussões do processo de saúde-doença.

O conceito de saúde englobada hodiernamente pela Psicologia da saúde é o formulado pela Organização Mundial de Saúde - OMS como o bem-estar físico, psíquico e social do indivíduo, e não somente como a ausência de uma doença ou de uma enfermidade (World Health Organization, 1999). É imperativo ressaltar esse conceito devido às novas diretrizes elaboradas pela
OMS para os programas e as políticas de atenção e de intervenção à saúde, quer primárias, quer secundárias ou terciárias, que não são mais focados no binômio saúde-doença. Nesse sentido, como afirmam Sebastiani e Maia (2005), a Psicologia da saúde tem sido considerada um campo de trabalho da Psicologia que nasce para dar resposta a uma demanda sociossanitária (por ir além de uma intervenção curativa ou terciária), pautada em um modelo biopsicossocial e ambiental (que resgata uma visão integral do indivíduo, não mais focalizado no processo de doença, compreendendo o binômio saúdedoença como um fenômeno multicausal e de inter-relação entre indivíduo-ambiente), sob uma perspectiva interdisciplinar (com a participação efetiva de outras áreas do saber na discussão e no exercício de atenção à saúde).

Nessa dimensão globalizante, com base nesse novo paradigma que norteia as ações de saúde, e sublinhando o caráter integrativo das várias visões sobre saúde, propõe-se a conexão dos aportes e das experiências da cronobiologia.

\section{Os desafios temporais: compreendendo a cronobiologia}

A cronobiologia é a disciplina que se ocupa da investigação temporal periódica, expressa em horas, dias, meses e/ou anos, de variáveis fisiológicas e comportamentais (ou seja, de ritmos biológicos) bem como de seus mecanismos e propriedades nos seres vivos. A ritmicidade biológica é de fundamental importância para a sobrevivência de uma espécie por promover ou auxiliar a adequação dos seus processos fisiológicos, das suas características anatômicas e das suas características comportamentais ao seu ambiente, que é rítmico e que é expresso ou em função do ciclo claro-escuro ou através 
Os ritmos

circadianos

são observados

em tarefas

relacionadas à

memória e ao

desempenho

cognitivo,

em variáveis

fisiológicas como

a temperatura

corporal, e na

alternância entre

a vigília e o sono

(Andrade, 1997). das estações do ano (Marques, Golombek, \& Moreno, 2003). Entretanto, mesmo se o ambiente apresentasse uma condição constante, como temperatura quente, por exemplo, a ritmicidade biológica continuaria a expressar-se durante dias, meses ou anos, dependendo das espécies, pois os ritmos biológicos demonstram caráter endógeno, independente da influência do ambiente (Marques et al., 2003).

A endogeneidade dos ritmos proporciona ao organismo a capacidade de dar respostas antecipatórias (modificações nas funções biológicas e comportamentais) às mudanças do ambiente, favorecendo a adaptação do organismo a essas mudanças, possivelmente pelas estruturas que permitem gerar essa ritmicidade. Como exemplo de modificações biológicas, temos o aumento na secreção de cortisol (hormônio relacionado com a preparação ao estado de alerta) no final do sono, que prepara o indivíduo para o início da vigília (Aschoff, Daan, \& Honma, 1982).

Em que pese a importância de se estudar a endogeneidade dos ritmos, o estudo da expressão da ritmicidade em condições naturais pode oferecer melhor entendimento dos mecanismos da sincronização. Dos ciclos ambientais mais evidentes, o ciclo claroescuro ou dia e noite é o mais importante para a sincronização dos ritmos, que consiste na manutenção do ajuste temporal entre o organismo e seu ambiente. Em função da organização fisiológica e comportamental e de sua relação com o dia e a noite, o ser humano é classificado como uma espécie diurna, ou seja, concentra suas atividades na fase clara ou diurna e o seu repouso (sono) na fase escura ou noturna. A expressão dos ritmos biológicos associados ao claro-escuro ou dia e noite são chamados de ritmos circadianos. Esses ritmos foram assim definidos por Halberg (1969) por apresentarem períodos em torno de 24 horas, sincronizados a ciclos ambientais de 24 horas.
O sistema de temporização circadiano (STC) é um conjunto de estruturas e mecanismos capaz de gerar o ritmo circadiano endogenamente através de uma estrutura neural: os núcleos supraquiasmáticos (NSQ). O NSQ é atualmente reconhecido tanto como o principal marcapasso dos mamíferos quanto como um sincronizador interno que permite diferentes níveis de sincronização dos ritmos fisiológicos do nosso organismo. $\mathrm{O}$ STC, além de sincronizar o ritmo com pistas temporais do ambiente (ordem temporal externa) ao ciclo das 24 horas (claro-escuro), mantém a ordem temporal interna (os ritmos de sistemas e órgãos de um organismo em relações de fases estáveis entre si). Como exemplo dessa ordem temporal interna, tem-se a coincidência no tempo dos valores mínimos da temperatura de nosso sangue arterial (temperatura central) com a fase de maior probabilidade de ocorrência do sono paradoxal, ou ainda a ocorrência de secreção do hormônio de crescimento durante o terço inicial da noite de sono (Almondes, 2007; Marques et al., 2003). Os ritmos circadianos são observados em tarefas relacionadas à memória e ao desempenho cognitivo, em variáveis fisiológicas como a temperatura corporal, e na alternância entre a vigília e o sono (Andrade, 1997).

Eventos ambientais periódicos como os horários de trabalho, de estudo, de lazer e outras atividades sociais são também pistas temporais ambientais, além do claroescuro, capazes de sincronizar o sistema de temporização circadiano no ser humano, mantendo a ordem temporal externa. Só que esses eventos sincronizadores podem entrar em conflito com a fase (momento determinado) de expressão do ritmo biológico. Os horários de trabalho são exemplos de fortes sincronizadores, pois os esquemas de trabalho em empresas/indústrias não se limitam apenas aos horários diurnos, mas estendem-se aos horários noturnos e aos finais de semana. Surge daí uma 
oposição entre os sincronizadores: primeiro, o horário de trabalho noturno faz com que o indivíduo desloque a atividade para a noite e o repouso para o dia, e, segundo, o horário de trabalho entra, também, em oposição aos horários sociais, pois os primeiros coincidem com os horários adotados para os afazeres domésticos, para o lazer e para os horários normais da sociedade (fortes sincronizadores) como, por exemplo, tarefas de casa, cuidados com os filhos, refeições com a família, tempo de lazer com os amigos, atividades sociais e funcionamento dos estabelecimentos sociais (Moreno, Fischer, \& Rotenberg, 2003; Monk, 2000).

Essa oposição entre a fase do sincronizador e a fase da expressão do ritmo biológico citado no exemplo de horários de trabalho ocasiona uma dessincronização externa. Todavia, isso também leva a uma dessincronização interna dos ritmos. Em função das mudanças nos sincronizadores (que, no caso citado, são os horários de trabalho na fase do escuro) há uma quebra da relação de fases dos ritmos biológicos com os sincronizadores ambientais (dessincronização externa) e uma quebra de relações de fase entre esses ritmos (dessincronização interna). O resultado é que os períodos das oscilações desses ritmos não se mantêm, e eles não se ajustam simultaneamente, alguns de forma mais rápida e outros mais lentos, às modificações externas (Almondes, 2007; Marques et al., 2003).

Todas essas perturbações são explicadas hoje pela natureza funcional do sistema de temporização circadiano, que é composto por multiosciladores. Esses osciladores possuem frequências diferentes, e, devido a isso, é possível ocorrer a sincronização. Durante uma determinada fase, esses osciladores tornam-se mais acoplados, expressando uma velocidade média, ou seja, eles estão agrupados. Em outra fase, os osciladores não estão acoplados, e, assim, cada oscilador apresenta sua própria velocidade, estando desagrupados. Pela existência de grupos de osciladores com velocidades diferentes, é possível que alguns ritmos sejam sincronizados mais rapidamente e outros, mais lentamente. Em situações de conflito entre o sistema de temporização circadiano e os sincronizadores ambientais, pode ocorrer uma dissociação na expressão da ritmicidade circadiana, fazendo com que um grupo de osciladores fique sincronizado com um sincronizador, como o claro-escuro, enquanto um outro grupo de osciladores sincroniza com um outro sincronizador, como o horário de trabalho, e/ou outro grupo pode expressar-se em livre curso (Cambras, Chiesa, Araújo, \& Díez-Noguera, 2004).

Dessa forma, a cronobiologia permite que se possa entender os desafios temporais que nos são impostos constantemente, ajudando a refletir sobre quais estratégias de enfrentamento se pode utilizar e se essas soluções conciliam nossa saúde biopsicossocial.

\section{O tempo na saúde e a saúde no tempo: conversas entre a Psicologia da saúde e a cronobiologia}

As dessincronizações internas e externas dos ritmos trazem uma série de consequências e de repercussões negativas para a saúde biopsicossocial e ambiental dos indivíduos. As consequências vão desde doenças relacionadas ao sistema gastrointestinal, como constipação, diarreia, indigestão e maior número de casos de úlcera péptica, até fadiga crônica, alterações de humor, distúrbios cardiovasculares, hipertensão, diminuições no nível de vigilância e efeitos negativos sobre o desempenho em tarefas que requerem atenção e concentração (Ferguson, Kennaway, Baker, Lamond, \& Dawson, 2012; Knutsson, 2004; Landsbergis, 2004; 
Costa (2004)

explica que o trabalho em turnos é estressante, e pode causar uma ativação neurovegetativa nos indivíduos, com maior secreção de hormônios do estresse e os consequentes efeitos no ritmo cardíaco, no metabolismo lipídico e nos processos trombóticos.
Monk, 2000; Moreno \& Louzada, 2004; Rotenberg, Portela, Marcondes, Moreno, \& Nascimento, 2001). Além disso, alguns dados de literatura relatam ausências dos indivíduos do trabalho por razões de saúde relacionadas às dessincronizações (Philip, Taillard, Niedhammer, Guilleminault, \& Bioulac, 2001) e maior número de visitas às clínicas médicas, com queixas de problemas relacionados à memória, à concentração, à atenção e à resolução de problemas (Kleiven, Boggild, \& Jeppesen, 1998; Zammit, Weiner, \& Damato, 1999). Algumas repercussões clássicas socioambientais negativas e graves são relatadas, como os acidentes com operadores de processos industriais, acidentes de trânsito e acidentes com aviação (Äkerstedt, 1994; Horne \& Reyner, 1995; Laundry \& Lees, 1991; Samel, Wegmann, \& Vejvoda, 1995), além dos mega-acidentes como o de Three-Mile Island (em 1979), o de Chernobyl (em 1986) e o de Exxon Valdez (em 1989) (Dinges, 1995; Moreno, Fisher, \& Menna-Barreto,2003; Ohayon, Lemoine, Arnaud-Briant, \& Dreyfus, 2002).

Esses efeitos lesivos, que são decorrentes da incompatibilidade temporal entre os ritmos de nosso organismo e as exigências de funcionamento de uma sociedade 24h, permeiam diversas instituições que apresentam uma organização temporal de suas atividades laborativas de forma contínua e ininterrupta, que vai além dos horários diurnos e ao longo dos dias estabelecidos normalmente como os de descanso semanal, como, por exemplo, os hospitais. Nesse contexto, os profissionais de saúde são atingidos pelos efeitos das dessincronizações. Dados de Borges e Fischer (2003) e de Fischer, Teixeira, Borges, Gonçalves e Ferreira (2002) mostraram que profissionais de saúde que trabalhavam em hospitais em um esquema de turno alternante de $12 \mathrm{~h}$ apresentavam diminuição da alerta quando em turno noturno. Vários trabalhos apontam que profissionais de saúde submetidos a esquema noturno ou alternante apresentam prejuízos cognitivos, fadiga, ansiedade, problemas emocionais, depressão e distúrbios de sono (Iskra-Golec, Folkard, Marek, \& Noworol, 1996; Saricaoğlu et al., 2005). Costa (2004) explica que o trabalho em turnos é estressante, e pode causar uma ativação neurovegetativa nos indivíduos, com maior secreção de hormônios do estresse e os consequentes efeitos no ritmo cardíaco, no metabolismo lipídico e nos processos trombóticos. Essas consequências representam um alto risco de acidentes e de erros relacionados ao trabalho, portanto, o conhecimento desses aspectos cronobiológicos pode aprovisionar os psicólogos da saúde, por mediação de programas de educação, para prevenir o aparecimento dessas doenças em profissionais de saúde.

Pacientes internados em hospitais também são acometidos pelas oposições entre sincronizadores. O ambiente hospitalar predispõe e perpetua as dessincronizações externas e internas, pois a própria estrutura física (barulho, ambiente muito frio ou muito quente, luz, colchão não apropriado) e a rotina de trabalho dentro de um hospital (interrupções no decorrer da noite para medicação) não é elaborada levando-se em conta que os pacientes necessitam de um padrão do ciclo sono e vigília (ritmo circadiano) regular e uma boa qualidade de sono para ajudar na sincronização. Dados de literatura revelam que, em situações de alterações de sono, há aumento da susceptibilidade a uma infecção. Lange, Perras, Fehm e Born (2003) mostraram que a privação de sono após a imunização contra hepatite $A$ reduziu significativamente $o$ número de anticorpos produzidos.

Além disso, quando o paciente é hospitalizado, há um acúmulo de tensões que enfraquecem temporariamente o equilíbrio funcional dos mecanismos adaptativos dos indivíduos em decorrência do fato de a situação ser 
encarada como nova, que exige respostas diferentes e desconhecidas das que ele está habituado a usar para solucionar seus problemas e que é geradora de sentimentos do medo da morte e do sofrimento que a doença ocasiona, de ansiedade, depressão e estresse. Em consequência, essa situação é denominada crise adaptativa, e age como fator que predispõe aos distúrbios de sono no indivíduo, pois tem um papel crítico na manutenção do despertar, levando a mais dessincronização, já que o indivíduo se mantém acordado na fase em que deveria estar dormindo, em função do alto nível de ansiedade e estresse. O resultado é que a frequência de transtornos do sono em pacientes internados, entre eles a insônia, pode ser até duas vezes maior se comparada à população geral, levando a maior tempo de internação e a maior uso indiscriminado de hipnóticos ao se receber a alta hospitalar (Freedman, Gazendam, Levan, Pack, \& Schwab, 2001; Parthasarathy \& Tobin, 2003).

Almondes e Araújo (2008), analisando a qualidade de sono de pacientes em hospitais públicos e privados, verificaram que pacientes de ambos os hospitais apresentavam qualidade de sono ruim, e que $70 \%$ em média de todos os pacientes apresentavam sonolência diurna excessiva, associada à interrupção do sono noturno para medicação. Essa discussão cronobiológica (dessincronizações e alterações de sono) é importante para a psiconeuroimunologia (uma subárea da Psicologia da saúde que integra a Psicologia, a neurologia e a imunologia), que trabalha calcada fundamentalmente na teoria do estresse, pois este gera a prevenção do alargamento da permanência do paciente no hospital, cogitando os fatores de risco apontados acima. Para se ter uma ideia, pacientes com insônia crônica apresentam redução das células relacionadas com o sistema imunológico -CD3, CD4 e CD8 (Savard, Laroche, Simard, Ivers, \& Morin, 2003).
O psicólogo da saúde/hospitalar trabalha com o entendimento e o tratamento dos aspectos psicológicos em torno do adoecimento, entendendo aqui que os aspectos psicológicos englobam comportamentos, pensamentos, crenças e estilo de vida, entre outros. O profissional também trabalha avaliando e fazendo o prognóstico dos comportamentos prejudiciais para a saúde, a fim de promover um comportamento saudável, e é treinado a dar assistência à pessoa enferma frente a uma situação de crise adaptativa, devendo dedicar particular atenção ao diagnóstico precoce dessa crise para ajudar o paciente no manejo das situações de crise. Empossado dos conhecimentos cronobiológicos, o psicólogo da saúde acrescenta mais um olhar aos seus objetivos, melhor compreensão do processo de adaptação não só psicológico mas também biológico do organismo a essas mudanças ambientais.

A Psicologia da saúde se beneficia em manter relação de interdisciplinaridade com a cronobiologia pelo conhecimento que esta propõe na compreensão da dimensão temporal de nosso organismo. A cronobiologia mostra que, em função das dessincronizações resultantes da oposição entre ritmos biológicos e demandas sociais, há alterações biopsicossociais. A Psicologia da saúde trabalha com o indivíduo no processo saúde-doença de forma holística, e a compreensão dessas dessincronizações e de suas consequências ajuda-a na identificação precoce de pessoas em situação de risco e na prevenção de comportamentos mais saudáveis para enfrentar ou para alterar essas situações.

A cronobiologia auxilia a Psicologia da saúde no seu papel de elaboração de programas efetivos de promoção de condutas saudáveis e de estudos e intervenções no campo comunitário e na saúde pública. A fim de contornar isso, tem sido sugerido um programa de higiene de sono (medidas 
comportamentais e ambientais que auxiliam e promovem um sono de boa qualidade) nos serviços hospitalares e a avaliação criteriosa das queixas de sono dos pacientes pela equipe médica. A adoção dessas medidas promoverá menor tempo de internação, redução do uso indiscriminado de hipnóticos e, em consequência, redução dos custos do tratamento. Por outro lado, também é importante orientar os profissionais de saúde quanto aos efeitos das dessincronizações sofridos por eles. O psicólogo da saúde, sensível à equipe de trabalho e objetivando o entendimento e o tratamento dos aspectos psicológicos em torno do adoecimento, poderá propor discussões acerca das percepções, crenças e estratégias para organizar os problemas de sono e para haver maior tolerância ao esquema de trabalho, em especial ao trabalho noturno, pois há trabalhos que mostram que as estratégias utilizadas nem sempre são adequadas, trazendo mais prejuízo à saúde física, mental e social. Além disso, o profissional poderá contribuir com proposições sobre a organização temporal das atividades e demandas nas instituições hospitalares junto à gestão hospitalar, a fim de evitar acidentes e erros.

No que se refere à medicação, outra interseção possível da Psicologia da saúde com a cronobiologia, como a primeira também atua em nível de intervenção terciária e a segunda colabora com o princípio segundo a qual o estado dos organismos varia regularmente ao longo do dia, ambas podem ajudar outros profissionais de saúde a valorizar a organização temporal da medicação. Dados da literatura mostram como a dosagem de drogas para induzir respostas satisfatórias e tratar estados patológicos tem eficácia diferencial segundo o horário do dia ou da noite e o momento da administração dos medicamentos aos pacientes (Moreno et al., 1999; Aguilar-Roblero et al., 2001).

\section{Considerações finais}

Como a qualidade de vida é resultado de uma inter-relação entre vários fatores, tais como: saúde física e saúde mental, longevidade, satisfação no trabalho, relações familiares, disposição, produtividade, dignidade e espiritualidade, que moldam e diferenciam o dia a dia do ser humano e resultam em uma rede de fenômenos, pessoas e situações (Minayo, Hartz, \& Buss, 2000; WHOQOL Group 1994 como citado em Fleck, 1998), pode-se concluir que o desafio que todos os indivíduos encontram é conciliar o tempo do organismo com as demandas temporais de uma sociedade que funciona $24 \mathrm{~h}$, sem tantas repercussões à saúde biopsicossocial. Cabe ao psicólogo da saúde, em seu papel de educador e de promotor em saúde, reconhecer a importância dos aspectos cronobiológicos em sua análise no trabalho com pacientes, profissionais e instituições de saúde, resgatando sempre seu papel de pesquisador básico e de intervencionista em saúde. 


\section{Katie Moraes de Almondes}

Doutora em Psicologia pela Universidade Federal do Rio Grande do Norte e docente da Universidade Federal do Rio Grande do Norte, Natal - RN - Brasil.

E-mail: katie.almondes@gmail.com

\section{Endereço para envio de correspondência:}

Campos Universitário, Caixa Postal 1622, Lagoa Nova. CEP: 59078-970. Natal, RN.

Recebido 01/12/2011, 1a Reformulação 04/04/2013, Aprovado 11/04/2013.

\section{Referências}

Aguilar-Roblero, R., Aréchiga, H., Ashkenazi, I., Burioka, N., Cipolla-Neto, J., Cornélissen, G., Markus, R., Marques, N., Menezes, A. A. L., Monk, T. H., Ralph, M.,Valdez-Ramirez, P., \& Menna-Barreto, L. (2001). The brain decade in debate: IV. Chronobiology. Brazilian Journal of Medical and Biological Research, 34, 831-841.

Äkerstedt, T. (1994). Work injuries and time of day-national data. Work hours, sleepiness and accidents. Stress Research Reports, 248, 106.

Almondes, K. (2007). Qualidade de sono e qualidade de vida em trabalhadores submetidos a diferentes esquemas de trabalho de uma empresa petroquímica (Tese de Doutorado). Universidade Federal do Rio Grande do Norte, Natal. Recuperado em 27 de maio, 2013 de ftp://ftp.ufrn.br/pub/ biblioteca/ext/bdtd/KatieMA.pdf

Almondes, K. M., \& Araújo, J. F. (2008). Sleep-wake cycle pattern, sleep quality and complaints about sleep disturbances made by inpatients. Sleep Science, 1, 35-39.

Andrade, M. M. M. (1997). Padrões temporais das expressões da sonolência em adolescentes. (Tese de Doutorado). Instituto de Ciências Biomédicas, Universidade de São Paulo, SP.
American Psychological Association. Division of Health Psychology 38. (2011). Health Psychology. Recuperado em março, 2013, de http://www.apa.org/about/division/div38.aspx

APA - Task Force on Health Research. (1976). Contributions of psychology to health research: Patterns, problems and potentials. American Psychologist, 31, 263-274.

Aschoff, J., Daan, S., \& Honma, K. I. (1982). Zeitgebers, entrainment and masking: Some unsettled questions. In J. Aschoff, S. Daan \& G. A. Gross (Eds.), Vertebrate circadian systems - structure and physiology (pp. 13-24). Berlim: Springer Verlag.

Borges, F. N. S., \& Fischer, F. M. (2003). Twelve-hour night shifts of healthcare workers: A risk to the patients? Chronobiology International, 20, 351-360.

Cambras, T., Chiesa, J., Araújo, J. F., \& DíezNoguera, A. (2004). Effects of photoperiod on rat motor activity rhythm at the lower limit of entrainment. Journal of Biological Rhythms, 19, 216-225.

Costa, G. (2004). Saúde e trabalho em turnos e noturno. In F. M. Fischer, C. Moreno \& L. Rotenberg. (Orgs.), Trabalho em 
turnos e noturno na sociedade 24 horas (pp. 79-98). São Paulo: Editora Atheneu.

Dinges, D. F. (1995). An overview of sleepiness and accidents. Journal Sleep Research., 4(Suppl. 2), 4-14.

Ferguson, S. A., Kennaway, D. J., Baker, A., Lamond, N., \& Dawson, D. (2012). Sleep and circadian rhythms in mining operators: Limited evidence of adaptation to night shifts. Applied Ergonomics, 43, 695-701.

Fischer, F. M., Teixeira, L. R., Borges, F. N. S., Gonçalves, M. B L., \& Ferreira, R. M. (2002). Percepção do sono: duração, qualidade e alerta em profissionais da área de enfermagem. Cadernos de Saúde Pública, 18, 1261-1269.

Fleck, M. P. A. (1998). Projeto desenvolvido para a OMS no Brasil pelo grupo de estudos em qualidade de vida no Depto. de Psiquiatria e Medicina Legal FAMED/UFRGS

Freedman, N. S., Gazendam, J., Levan, L., Pack, A. I., \& Schwab, R. J. (2001). Abnormal sleep/wake cycles and the effect of environmental noise on sleep disruption in the intensive care unit. American Journal of Respiratory and Critical Care Medicine, $163,451-57$.

Halberg, F. (1969). Chronobiology. Annual Review of Physiology, $31,675-725$.

Horne, J. A., \& Reyner, L. A. (1995). Driver sleepiness. Journal Sleep Research, 4(Suppl. 2), 23-29.

Iskra-Golec, I., Folkard, S., Marek, T., \& Noworol, C. (1996) Health, well being and burnout of ICU nurses on 12- and 8-h shifts. Work Stress, 10(3), 251-256.

Kleiven, M., Boggild, H., \& Jeppesen, H. J. (1998). Shift work and sick leave. Scandinavian Journal Work Environment Health, 24(Suppl. 3), 128-133.

Knutsson, A. (2004). Methodological aspects of shift-work research. Chronobiology International, 21, 1049-1053.

Landsbergis, P. (2004). Long work hours, hypertension, and cardiovascular disease. Cadernos de Saúde Pública, 20, 1746-1748.

Lange, T., Perras, B., Fehm, H. L., \& Born, J. (2003). Sleep enhances the human antibody responses to hepatitis $A$ vaccination. Psychosomatic Medicine, 65, 831-835.

Laundry, B. R., \& Lees, R. E. M. (1991). Industrial accident experience of one company on 8-and 12-hour shifts systems. Journal of Occupational Medicine, 33, 903-906.

Marques, M. D., Golombek, D., \& Moreno, C. (2003). Adaptação temporal. In N. Marques \& L. Menna-Barreto. (Orgs.), Cronobiologia: princípios e aplicações (pp. 55-98). São Paulo: Fiocruz /Edusp.

Matarazzo, J. D. (1980). Behavioral health and behavioral medicine: Frontiers for a new health psychology. American Psychologist, 35, 807-817.

Minayo, M. C. S., Hartz, Z. M. A., \& Buss, P. M. (2000) Qualidade de vida e saúde: um debate necessário. Ciência \& Saúde Coletiva, 5, 7-31.

Monk, T. H. (2000). Shift work. In M. H. Kryger, T. Roth \& W.C. Dement (Eds.), Principles and practice of sleep medicine (pp. 521- 525). United States of America: W. B. Saunders Company.

Moreno, C., Fisher, F. M., \& Menna-Barreto, L. (2003). Aplicações da cronobiologia. In N. Marques \& L. Menna-Barreto (Orgs.), Cronobiologia: princípios e aplicações (pp. 259-264). São Paulo: Fiocruz / Edusp.

Moreno, C., Fischer, F. M., \& Rotenberg, L. (2003). A saúde do trabalhador na sociedade 24 horas. São Paulo em Perspectiva, $17(1), 34-46$

Moreno, C., \& Louzada, F. M. (2004). What happens to the body when one works at night? Cadernos de Saúde Pública, 20, 1739-1745.

Ohayon, M. M., Lemoine, P., Arnaud-Briant, V., \& Dreyfus, M. (2002). Prevalence and consequences of sleep disorders in a shift worker population. Journal of Psychosomatic Research, $53,577-583$.

Parthasarathy, S., \& Tobin, M. J. (2003). Is sleep disruption related to severity of critical illness? American Journal of Respiratory and Critical Care Medicine, 167, 968-74.

Philip, P., Taillard, J., Niedhammer, I., Guilleminault, C., \& Bioulac, B. (2001). Is there a link between subjective daytime somnolence and sicknes absenteeism? A study in a working population. Journal Sleep Research, 10, 111-115.

Rotenberg, L., Portela, L. F., Marcondes, W. B., Moreno, C., \& Nascimento, C. P. (2001). Gênero e trabalho noturno: sono, cotidiano e vivências de quem troca a noite pelo dia. Cadernos Saúde Pública, 17, 639-649.

Samel, A., Wegmann, M., \& Vejvoda, M. (1995). Jet lag and sleepiness in aircrew. Journal of Sleep Research, 4(Suppl. 2), 30-36.

Saricaoğlu, F., Akinci, S. B., Gözaçan, A., Güner, B. Rezaki, M., \& Aypar, U. (2005). The effect of day and night shift working on the attention and anxiety levels of anesthesia residents. Turkish Journal of Psychiatry, 16, 106-112.

Savard, J., Laroche, L., Simard, S., Ivers, H., \& Morin, C. M. (2003). Chronic insomnia and immune functioning. Psychosomatic Medicine, 65, 211-221.

Sebastiani, R. W., \& Maia, E. M. C. (2005). Contribuições da psicologia da saúde - hospitalar na atenção ao paciente cirúrgico. Acta Cirurgica Brasileira, 20(Supl. 1), 50-55. doi: http://dx.doi.org/10.1590/S0102-86502005000700010

Westphal, M. F., \& Santos, J. L. F. (1999). Práticas emergentes de um novo paradigma de saúde: o papel da universidade. Estudos Avançados, 13(35), 71-88.

World Health Organization - OMS. Division of Child Health and Development (CHD). (1999). Recuperado em 02 novembro, 2011, de htpp://www.who.org.nov

Zammit, G. K., Weiner, J., \& Damato, N. (1999). Quality of life in people with insomnia. Sleep, 22, S379-S385. 